\title{
Novel applications of plasmonics and photonics devices to sub-THz wireless
}

Maurizio Burla, Claudia Hoessbacher, Wolfgang Heni, Christian Haffner, Yuriy Fedoryshyn, et al.

Maurizio Burla, Claudia Hoessbacher, Wolfgang Heni, Christian Haffner, Yuriy Fedoryshyn, Dominik Werner, Tatsuhiko Watanabe, Yannick Salamin, Hermann Massler, David Hillerkuss, Delwin Elder, Larry Dalton, Juerg Leuthold, "Novel applications of plasmonics and photonics devices to subTHz wireless," Proc. SPIE 11307, Broadband Access Communication Technologies XIV, 113070 I (31 January 2020); doi: 10.1117/12.2550323 


\title{
Novel applications of plasmonics and photonics devices to sub-THz wireless
}

\author{
Maurizio Burla ${ }^{\mathrm{a},{ }^{,},}$Claudia Hoessbacher ${ }^{\mathrm{a}}$, Wolfgang Heni ${ }^{\mathrm{a}}$, Christian Haffner, ${ }^{\mathrm{a}, \mathrm{b}, \mathrm{c}}$, \\ Yuriy Fedoryshyn ${ }^{\mathrm{a}}$, Dominik Werner ${ }^{\mathrm{a}}$, Tatsuhiko Watanabe ${ }^{\mathrm{a}}$, Yannick Salamin ${ }^{\mathrm{a}, \mathrm{d}}$, \\ Hermann Massler ${ }^{\mathrm{e}}$, David Hillerkuss ${ }^{\mathrm{f}}$, Delwin Elder ${ }^{\mathrm{g}}$, Larry Dalton ${ }^{\mathrm{g}}$, Juerg Leuthold ${ }^{\mathrm{a}}$ \\ anstitute of Electromagnetic Fields, ETH Zurich, Gloriastrasse 35, Zurich 8092, Switzerland; \\ ${ }^{\mathrm{b}}$ Maryland Nanocenter, University of Maryland, College Park, MD 20742 USA; ${ }^{\text {Physical }}$ \\ Measurement Laboratory, National Institute of Standards and Technology, 10 Gaithersburg, \\ Maryland 20899, USA; ${ }^{\mathrm{d}}$ Research Laboratory of Electronics, Massachusetts Institute of Technology, \\ Cambridge, Massachusetts 02139, USA; ${ }^{\mathrm{e}}$ Fraunhofer IAF, Tullastraße 72, 79108 Freiburg im \\ Breisgau, Germany; ${ }^{\mathrm{f}}$ Optical \& Quantum Laboratory, Huawei Technologies Duesseldorf GmbH I \\ Address: Riesstrasse 25-C3, 80992 München, Germany; ${ }^{\circ}$ Department of Chemistry, University of \\ Washington, Seattle, WA 98195-1700, USA \\ *maurizio.burla@ief.ee.ethz.ch
}

\begin{abstract}
We recently demonstrated modulators based on plasmonic technology displaying a flat frequency response reaching $500 \mathrm{GHz}$, high linearity and power handling. We discuss their potential for extending microwave photonics (MWP) applications to the sub- $\mathrm{THz}$ range, demonstrating analog photonic links with bandwidth in excess of $100 \mathrm{GHz}$ and the capability of direct mm-wave to optical conversion.
\end{abstract}

Keywords: Modulators, plasmonics, microwave photonics, analog links, radio-over-fiber, terahertz.

\section{INTRODUCTION}

Wireless data traffic has been growing exponentially, doubling every 22 months [1], and capacity demand is reaching tens to hundreds of $\mathrm{Gb} / \mathrm{s}$ [2]. To reach this speed target, large transmission bandwidths are required. The currently used microwave bands have a limited allocated spectrum, typically limited to a few hundred MHz. While millimetre-wave bands in the $60 \mathrm{GHz}$ and the 70-95 GHz ranges offer more spectrum, contiguous bandwidths are still below $9 \mathrm{GHz}$, which may be insufficient to support wireless data rates at hundreds of $\mathrm{Gb} / \mathrm{s}$ without drastically reducing link lengths [2]. As a consequence, some consider the $\mathrm{THz}$ band $(300 \mathrm{GHz}-10 \mathrm{THz})$ as the "next frontier" for future data links (Fig. 1a) due to the extremely large bandwidths available [3]. Accessing the $\mathrm{THz}$ spectrum, however, poses several challenges compared to microwaves, namely sensitivity to blockage, largely increased free-space path loss and atmospheric absorption due to water vapour [2].

In addition, the generation and detection of $\mathrm{THz}$ signals is not obvious; the most common approaches are either by means of electronic devices $[4,5]$ or photonic techniques [3, 6-8]. The latter approach is attractive as it offers the possibility of seamless integration with the existing fiber networks [6,9]. For optical-to-THz conversion, uni-travelling carrier photodiodes (UTC-PD) are a well-proven solution [3]. The creation of a complete THz link, however, also requires THz-to-optical conversion, which, in turn, demands very fast electro-optic modulators, with electro-optic bandwidth well above $300 \mathrm{GHz}$, high power handling and high linearity [10], Fig. 1b. Impressive progress has been reported in lithium niobate modulators [11], with demonstrations showing losses as low as $0.5 \mathrm{~dB}$ and an ultra-low RF half-wave voltage of $1.4 \mathrm{~V}$ [12], or measurable electro-optical response up to $500 \mathrm{GHz}$ [13]. Very recently, we showed a plasmonic-organic hybrid Mach-Zehnder modulator (POH-MZM) displaying the desired characteristics of speed, linearity and power handling simultaneously [14], with a flat frequency response up to $500 \mathrm{GHz}$, a third-order input 


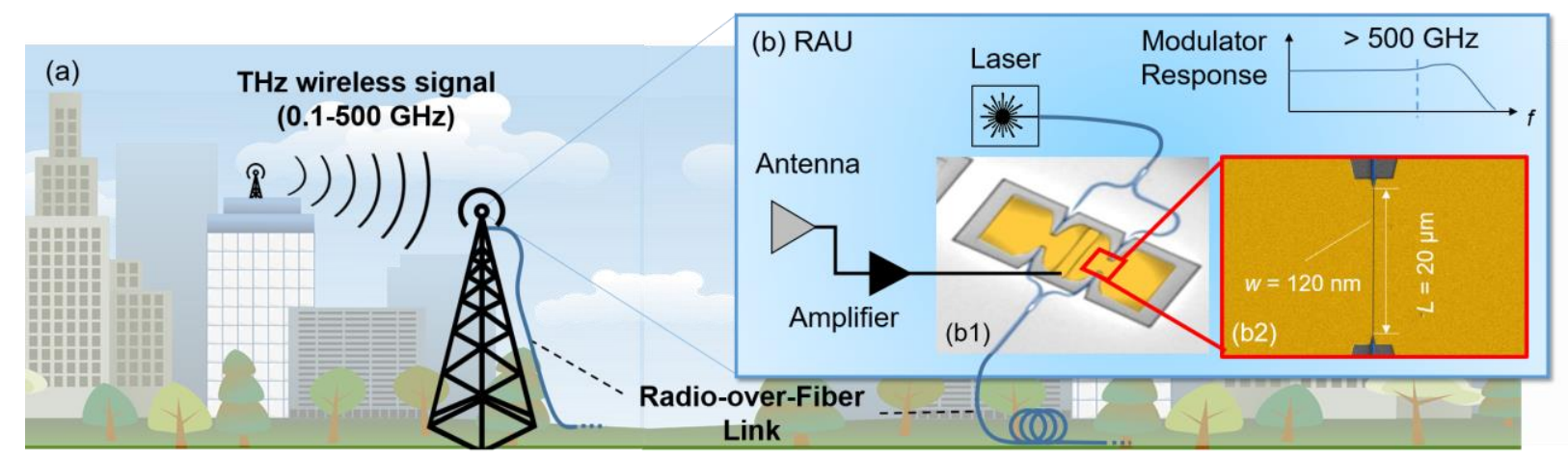

Figure 1. (a) A THz wireless communication scenario. (b) At the remote antenna unit (RAU), THz wireless signals are received by an antenna and converted to the optical domain to be transported over a radio-over-fiber analog link. A modulator with sub- $\mathrm{THz}$ bandwidth, high linearity and high-power handling transfers the $\mathrm{THz}$ signal onto an optical carrier with high fidelity. Inset: (b1) micrograph of a plasmonic Mach-Zehnder modulator with a plasmonic phase modulator in each arm; (b2) image of the $120 \mathrm{~nm}$-wide, $20 \mu \mathrm{m}$-long phase modulator slot waveguide. Image edited from [14].

intercept point (IIP3) of $18.9 \mathrm{dBm}$ and operation at high electrical power level up to $24.4 \mathrm{dBm}$, only limited by the power of the available RF amplifiers. In this paper, we present its characterization and applications to microwave photonic links with $>100 \mathrm{GHz}$ bandwidth and plasmonic mixers for direct wireless to optical conversion.

\section{PLASMONIC MODULATORS}

\subsection{Operating principle and device under test}

To build modulators with operation frequencies reaching the sub-THz range we use structures capable of guiding and processing surface plasmon polaritons (SPP). SPPs are electromagnetic surface waves existing at the interface between a metal and an insulator [15]. The guiding structure consists of a metallic slot waveguide, having a width of a few tens of nanometres, created between two metal electrodes, as visible in Fig. 1(b2). Light is fed to the slot where it excites the SPP using a tapered silicon waveguide. When the slot is filled with an organic material displaying second-order optical nonlinearity [16], an electric field applied between the electrodes will induce a linear variation of the material refractive index by means of Pockels effect. This structure therefore implements an electro-optic phase modulator. High modulation efficiency is achieved thanks to the strong light confinement in the slot as well as the excellent overlap between the optical and the electrical field profiles. This, in turn, allows to keep the modulator short (few tens of micrometres) which limits the SPP propagation losses. In addition, such modulator displays very high speeds thanks to its very low RC constant; the high conductivity of the metal electrodes yields sub- $\Omega$ resistances, and the small slot height (approx. $150 \mathrm{~nm}$ ) and length (few tens of micrometres) keeps capacitance to the femtofarad range, yielding cut-off in the THz range. To keep parasitic capacitances low, the metallic electrical pads of the modulator are kept as small as possible (while still allowing electrical contact to the device using a probe with $50 \mu \mathrm{m}$ pitch) and a ground-signal-ground configuration. Further details on the electrical modelling of the modulator are available in [17].

An intensity modulator is created by placing two phase modulators in an interferometer structure. In detail, the device under tests in Fig. 1(b1) includes one $20 \mu \mathrm{m}$-long POH phase modulator in each arm, Fig. 1(b2). Each phase modulator uses a $120 \mathrm{~nm}$-wide slot and the metal-insulator-metal (MIM) waveguide is filled with the organic electro-optic material HD-BB-OH/YLD124 [18]. The silicon linear tapers coupling light to and from the slot [19] are 1 micron-long and display a loss of approximately $1.1 \mathrm{~dB}$ each. Grating coupler losses are in the order of $5 \mathrm{~dB} /$ coupler. By cut-back measurement, losses in the plasmonic waveguide amount to approximately $0.5 \mathrm{~dB} / \mu \mathrm{m}$.

\subsection{Frequency response and linearity}

In view of application to analog radio-over-fiber links, we performed experiments to characterize relevant characteristics such as frequency response, nonlinear distortions and power handling of the POH-MZM.

The frequency response was tested over the range from $75 \mathrm{MHz}$ up to $500 \mathrm{GHz}$, limited by the available test equipment. A continuous-wave $(\mathrm{CW})$ laser at a wavelength of $1547.5 \mathrm{~nm}$ with $0 \mathrm{dBm}$ optical power was fed into the POH-MZM. 
We used two different setups to generate modulating carriers in the frequency ranges $15-70 \mathrm{GHz}$ and $200-500 \mathrm{GHz}$. In the lower frequency range, the signal was generated by electrical means. In the frequency range between 200 and $500 \mathrm{GHz}$, the test signal was generated optically by heterodyning two tuneable laser sources on a UTC-PD to produce a $\mathrm{THz}$ signal at a frequency equal to the difference between the ones of the two lasers. An optical spectrum analyser (OSA) is used to measure the amplitude ratio between the optical carrier and the first sidelobe. The resulting frequency response is displayed in Fig. 2(a) and shows a flat response over the complete $75 \mathrm{MHz}$ to $500 \mathrm{GHz}$ range, and the response never crosses the $-3 \mathrm{~dB}$ line compared to the low-frequency value. For comparison, the grey lines in the same figure display the responses measured in a previous experiment by Hoessbacher et al. in [20], who obtained them with a similar POH-MZM device. Complete details on the experimental setups and the procedure used to measure the modulator response are reported in [14].

Modulator linearity is a crucial parameter in all analog applications because it affects the system spurious-free dynamic range (SFDR) directly $[10,21]$. The SFDR is a quantitative indication of the realistic power range over which a two-port network is capable of operating in a linear regime without being limited by noise [10]. While noise poses a lower bound to this power range, nonlinear distortions (e.g. generation of spurious tones), determine the upper bound. A typical way of probing nonlinear distortions is the method known as two-tone test [21]. With this technique we have characterized the second- and third-order intermodulation distortions (IMD2 and IMD3), which limit the SFDR in sub-octave and multi-octave conditions, respectively [21]. In detail, we have compared the input intercept point of the POH-MZM with the one of a commercial modulator with the same half-wave voltage ( $\mathrm{U}^{2} \mathrm{~T}$ model MZMO2120: $V_{\pi}=3 \mathrm{~V}$, electro optical response of $33 \mathrm{GHz}$ at $3 \mathrm{~dB}$, typical insertion loss of $5 \mathrm{~dB}$, typical extinction ratio of $23 \mathrm{~dB}$ ), see Fig. 2(b-c). We obtained a value of $18.9 \mathrm{dBm}$, versus a value of $19 \mathrm{dBm}$ for the commercial device. This result proves that the the intermodulation distortions from the POH-MZM are as low as those obtained from a commercial modulator optimized for high-performance analog applications, with the same half-wave voltage.

(a)
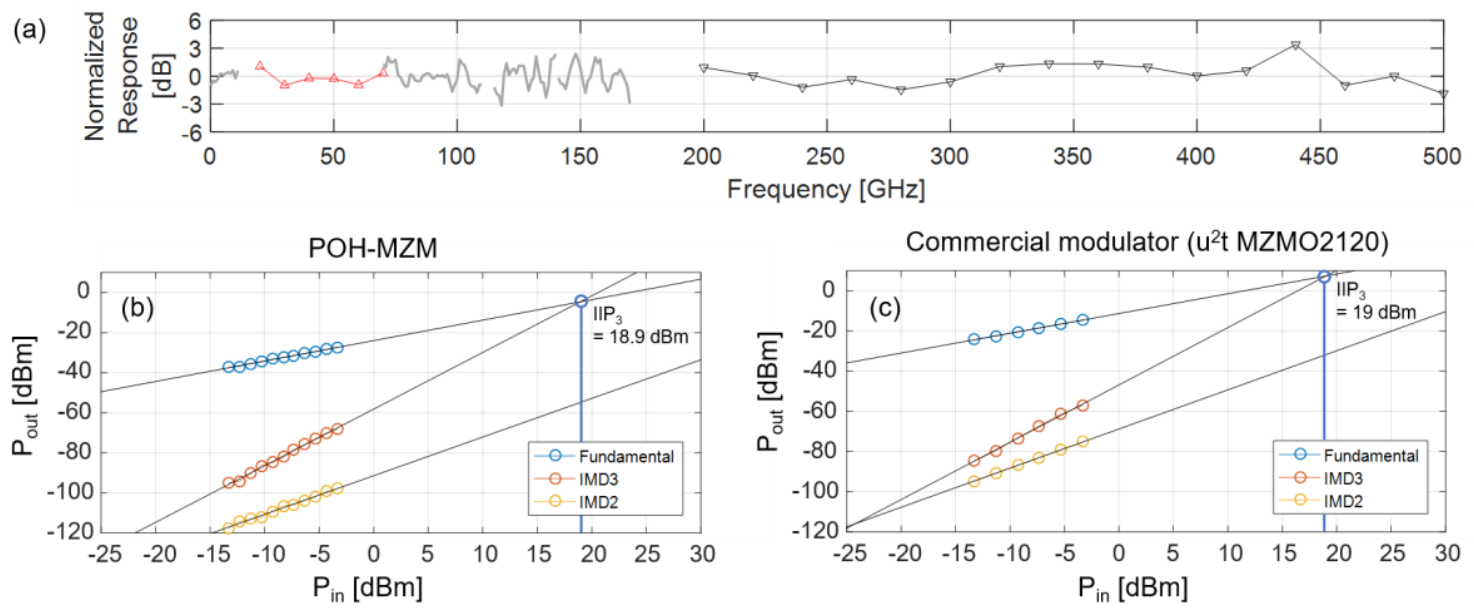

Figure 2. (a) Measured response of the $\mathrm{POH}-\mathrm{MZM}$ over the frequency range $75 \mathrm{MHz}-500 \mathrm{GHz}$. The figure is obtained normalizing the measured power of the optical modulation sidebands, to the electrical power available at the input of the POH-MZM. The device features a flat response up to $500 \mathrm{GHz}$. (b-c) Second-order (IMD2) and third-order (IMD3) intermodulation distortions for the POH-MZM (a) and a commercial GaAs modulator (b). The horizontal axis indicates the available RF power at the modulator input generated by the source. The third-order input intercept point for the $\mathrm{POH}-\mathrm{MZM}$ is at $18.9 \mathrm{dBm}$, very close to the $19.0 \mathrm{dBm}$ obtained with a commercial high-performance GaAs modulator with the same half-wave voltage. Image edited from [14]. 


\section{APPLICATIONS}

\subsection{Sub-mm-wave analog photonic links}

A possible application enabled by the modulator in Fig. 1 is the creation of analog Radio-over-Fiber (RoF) links at sub$\mathrm{THz}$ frequencies. We have implemented such link as shown in Fig. 3(a-b), demonstrating direct THz-to-optical-to-THz conversion over $>100 \mathrm{GHz}$ bandwidth (220-325 GHz range), only limited by our electrical test equipment.
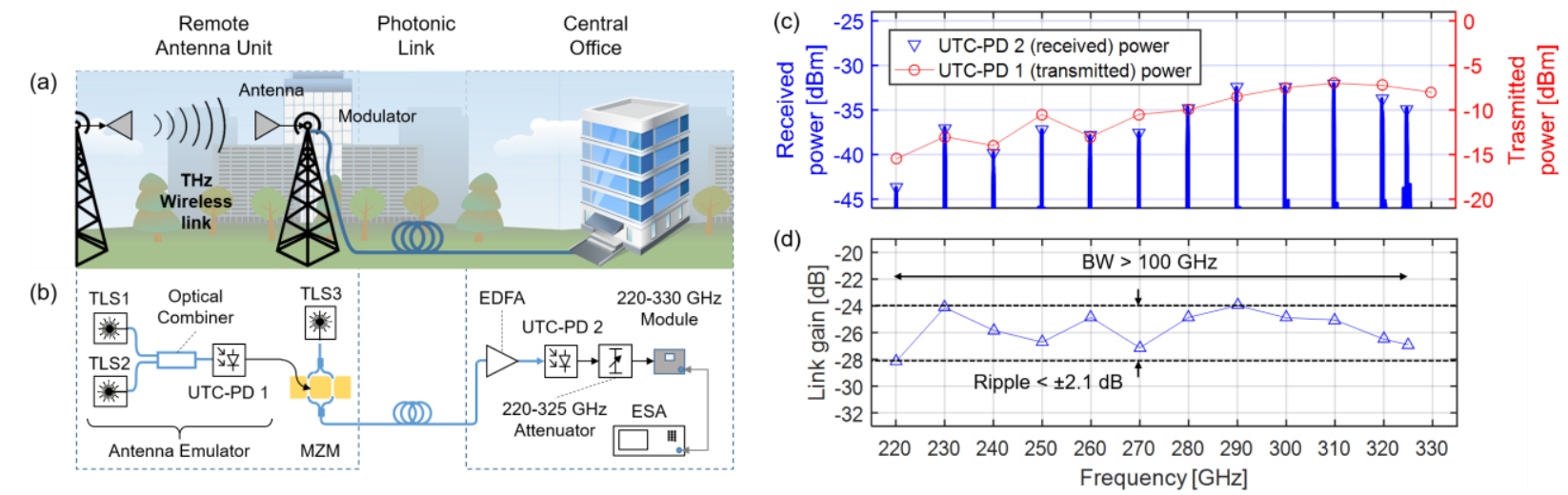

Figure 3. (a) Photonic link scenario connecting a remote antenna to a central office. (b) Experimental setup. The POHMZM modulates an optical carrier $f_{\text {carrier }}$ with a mm-wave signal in the $220-325 \mathrm{GHz}$ frequency range generated by UTC-PD 1. The modulated carrier is transported on a single-mode fiber (SMF), is amplified by an erbium-doped fiber amplifier (EDFA) and is received by UTC-PD 2. The detected mm-wave is analyzed with an electrical spectrum analyzer (ESA) using a mm-wave extension module. (c) Plasmonic organic hybrid Mach-Zehnder modulator; (d) falsecolor scanning-electron microscope image of a plasmonic phase modulator. Image edited from [14].

The signal to be transmitted along the link is generated using UTC-PD 1, emulating the antenna source in Fig. 3(a), and are used to directly modulate an optical carrier using a POH-MZM, Fig. 1(c). The modulated signal, after propagation through the single-mode fiber link, is amplified using a low-noise erbium-doped fiber amplifier (EDFA). The mm-waves are then detected with another UTC-PD (UTC-PD2) and are analysed using an electrical spectrum analyser (ESA) equipped with a mm-wave extension module. The transmitted (red circles) and received power spectrum (blue triangles) are displayed in Fig. 3(c). The link gain is obtained as the ratio of these two quantities and is plotted in Fig. 3(d). The link features a flat gain response over the complete $220-325 \mathrm{GHz}$ frequency range. A ripple of $\pm 2.1 \mathrm{~dB}$ across the band is attributed to ripples in the attenuator loss, which could not be tested in our laboratory at the time of the experiment.

The noise figure of an analog link can be written as [10]

$$
\mathrm{NF}=10 \log _{10}\left(\frac{P_{\mathrm{N}}}{g k T B}\right)
$$

where $P_{\mathrm{N}}$ is the total noise power dissipated on the load. In a basic externally-modulated photonic link, composed by a Mach-Zehnder modulator, a fiber link, and EDFA and a photodetector, the total link noise power is given by the sum of different noise terms:

$$
P_{\mathrm{N}}=(1+g) P_{\mathrm{th}}+\frac{1}{4} P_{\text {shot }}+\frac{1}{4} P_{\text {rin }}+\frac{1}{4} P_{\mathrm{EDFA}}
$$

The NF can be calculated to be link to be approximately $45.8 \mathrm{~dB}$ at $300 \mathrm{GHz}$. Improving the fiber-to-chip coupling loss from the current $5 \mathrm{~dB}$ to state-of-the-art values near $2.5 \mathrm{~dB}$, the overall noise figure of the link improves from $45.8 \mathrm{~dB}$ to $40.3 \mathrm{~dB}$ and the link gain improves from $-24 \mathrm{~dB}$ to $-14 \mathrm{~dB}$. 
Based on the noise and linearity characterization described in the previous section, it is possible to determine the spurious-free dynamic range of the analog optical link, as shown in Fig. 4. For the analog link test, we used a modulator with a higher $V_{\pi, \mathrm{RF}}(6.7 \mathrm{~V}$ instead of $3 \mathrm{~V})$ and a lower loss compared to the one used in the nonlinear distortion test. The power of the third order intermodulation terms (IMD3) reduces as the sixth power of $V_{\pi, \mathrm{RF}}$ and as the second power of the loss $L$, according to the equation [10,22], yielding a reduction of IMD3 power of $34.5 \mathrm{~dB}$. This results in an IIP3 of $38.1 \mathrm{dBm}$ and an SFDR3 of $105.2 \mathrm{~dB} / \mathrm{Hz}^{2 / 3}$, as shown in Fig. 3. Similarly, the IMD2 power reduces by the fourth power of $V_{\pi, \mathrm{RF}}$ and as the second power of the loss $L$, by $27.6 \mathrm{~dB}$, and the SFDR2 increases to $109.48 \mathrm{~dB} / \mathrm{Hz}^{1 / 2}$, as shown in Fig. 3. A detailed analysis of the performance metrics of this link (link gain, noise figure and spurious free dynamic range) are reported in [14].

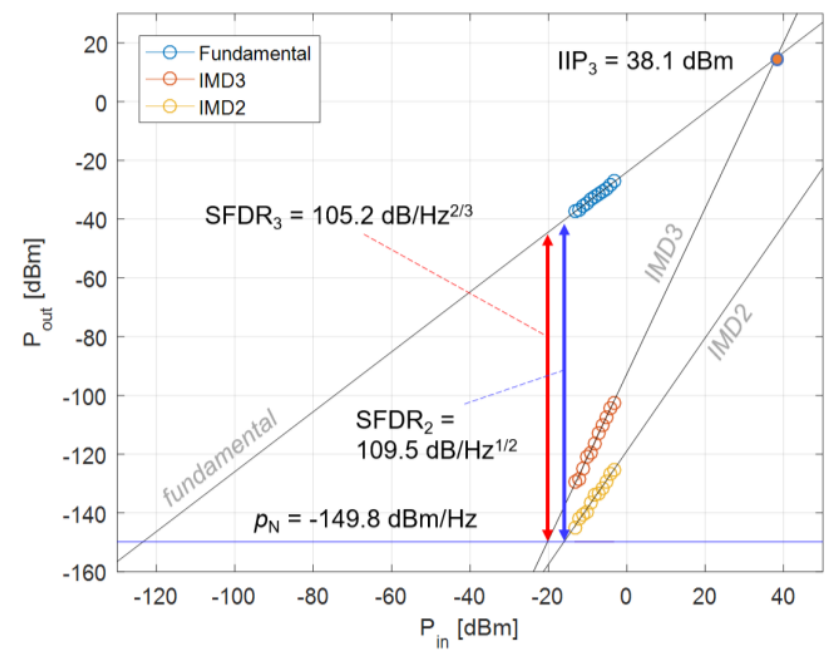

Figure 4. Estimation of spurious-free dynamic range of the $325 \mathrm{GHz}$ analog photonic link. Edited from [14].

\subsection{Plasmonic mixers}

Seamless integration of fiber and wireless networks at millimetre and terahertz frequencies demands for high-speed and efficient optical-to-wireless and wireless-to-optical converters. Wireless-to-optical conversion is generally achieved through electrical amplification and mixing followed by modulation. This normally requires separate electrical and optical high-speed components, which are complex and whose interfacing is not trivial at those high speeds. Recent efforts demonstrated Mach-Zehnder modulators directly driven by arrays of patch antennas and polarization-reversed structures, capable of converting the wireless signal to a single-sideband optical signal in the $60 \mathrm{GHz}$ band [23].

Recently, Salamin et al. successfully transmitted 20 and $10 \mathrm{Gbit} / \mathrm{s}$ over wireless distances of 1 and $5 \mathrm{~m}$, respectively, at a carrier frequency of $60 \mathrm{GHz}$, and converted them to an optical signal using a plasmonic phase modulator directly integrated with a resonant four-leaf-clover (4LC) antenna [9]. Such result could be demonstrated without resorting to any high-speed amplification or frequency conversion electronics in the front-end. This result is made possible by the inherent built-in plasmonic field enhancement (up to a factor 90,000) enabled by the plasmonic mixer. In addition, the complete electro-optic device has a footprint of only $0.315 \mathrm{~mm}^{2}$. Compared to previous approaches, the plasmonic one allows straightforward extension to the $\mathrm{THz}$ frequencies. The same author proposed and demonstrated a similar plasmonic mixer capable of operation in the $300 \mathrm{GHz}$ band [24]. Very recently, it was also shown how the same plasmonic technology can enable new broadband and ultra-efficient $\mathrm{THz}$ field detectors for $\mathrm{THz}$ time-domain spectroscopy [25]. The demonstrated plasmonic detector showed an electro-optic response up to $2.5 \mathrm{THz}$, confirming that plasmonics is could be an ideal solution for high-speed applications, especially at $\mathrm{THz}$ frequencies where no other technology was shown yet. 


\section{ACKNOWLEDGMENTS}

The authors acknowledge funding from the EU projects PLASILOR, PlasmoFab and PlaCMOS, the US National Science Foundation (DMR-1303080) and the Air Force Office of Scientific Research (FA9550-15-1-0319). M. Burla also acknowledges the SNF Ambizione grant nr. 173996.

\section{REFERENCES}

[1] T. Harter, S. Ummethala, M. Blaicher et al., "Wireless THz link with optoelectronic transmitter and receiver," (2019).

[2] A. J. Seeds, H. Shams, M. J. Fice et al., "TeraHertz Photonics for Wireless Communications," Lightwave Technology, Journal of, 33(3), 579-587 (2015).

[3] S. Jia, X. Pang, O. Ozolins et al., "0.4 THz Photonic-Wireless Link With 106 Gb/s Single Channel Bitrate," Journal of Lightwave Technology, 36(2), 610-616 (2018).

[4] [Keysight Technologies, mm-wave Source Modules].

[5] [Virginia Diodes, Signal Generator Extension Modules].

[6] T. Nagatsuma, S. Horiguchi, Y. Minamikata et al., "Terahertz wireless communications based on photonics technologies," Optics Express, 21(20), 23736-23747 (2013).

[7] L. K. Oxenløwe, S. Jia, X. Pang et al., "100s Gigabit/s THz Communication," OSA Technical Digest (online). STu3D.1.

[8] Y. Salamin, P. Ma, et al., "100 GHz Plasmonic Photodetector," ACS Photonics, 5(8), 3291-3297 (2018).

[9] Y. Salamin, B. Baeuerle, W. Heni et al., "Microwave plasmonic mixer in a transparent fibre-wireless link," Nature Photonics, 12(12), 749-753 (2018).

[10] C. H. Cox, [Analog optical links: theory and practice] Cambridge University Press, (2006).

[11] P. O. Weigel, J. Zhao, K. Fang et al., "Bonded thin film lithium niobate modulator on a silicon photonics platform exceeding $100 \mathrm{GHz} 3-\mathrm{dB}$ electrical modulation bandwidth," Optics Express, 26(18), 23728-23739 (2018).

[12] C. Wang, M. Zhang, X. Chen et al., "Integrated lithium niobate electro-optic modulators operating at CMOScompatible voltages," Nature, 562(7725), 101-104 (2018).

[13] A. J. Mercante, S. Shi, P. Yao et al., "Thin film lithium niobate electro-optic modulator with terahertz operating bandwidth," Optics Express, 26(11), 14810-14816 (2018).

[14] M. Burla, C. Hoessbacher, W. Heni et al., "500 GHz plasmonic Mach-Zehnder modulator enabling sub-THz microwave photonics," APL Photonics, 4, 1-11 (2019).

[15] C. Haffner, W. Heni, Y. Fedoryshyn et al., "Plasmonic Organic Hybrid Modulators - Scaling Highest Speed Photonics to the Microscale," Proceedings of the IEEE, 104, 2379 (2016).

[16] R. W. Boyd, [Nonlinear optics] Academic Press, (2003).

[17] C. Hoessbacher, [Plasmonic Switches and Modulators for Optical Communications] ETH Zurich, Zurich, Switzerland(2017).

[18] D. L. Elder, C. Haffner, W. Heni et al., "Effect of Rigid Bridge-Protection Units, Quadrupolar Interactions, and Blending in Organic Electro-Optic Chromophores," Chemistry of Materials, 29(15), 6457-6471 (2017).

[19] C. Haffner, W. Heni, Y. Fedoryshyn et al., "Plasmonic Organic Hybrid Modulators - Scaling Highest Speed Photonics to the Microscale," Proceedings of the IEEE, 104(12), 2362-2379 (2016).

[20] C. Hoessbacher, A. Josten, B. Baeuerle et al., "Plasmonic modulator with > $170 \mathrm{GHz}$ bandwidth demonstrated at 100 GBd NRZ," Optics Express, 25(3), 1762-1768 (2017).

[21] E. I. Ackerman, and C. H. Cox, "RF fiber-optic link performance," IEEE Microw. Magazine, 2(4), 50-58 (2001).

[22] D. A. I. Marpaung, [High Dynamic Range Analog Photonic Links: Design and Implementation] Twente University Press (TUP), Enschede, The Netherlands(2009).

[23] Y. Matsukawa, T. Inoue, H. Murata et al., "Millimeter-wave band optical single-sideband modulator using arrayantenna-electrode and polarization-reversed structures with asymmetric Mach-Zehnder waveguide," Japanese Journal of Applied Physics, 57(8S2), 08PB04 (2018).

[24] Y. Salamin, T. Blatter, Y. Horst et al., "300 GHz Plasmonic Mixer," Proc. 2019 International Topical Meeting on Microwave Photonics (MWP). 1-4.

[25] Y. Salamin, I.-C. Benea-Chelmus, Y. Fedoryshyn et al., "Compact and ultra-efficient broadband plasmonic terahertz field detector," Nature Communications, 10(1), 5550 (2019). 